\title{
Non-Newtonian Effects on the Squeeze Film Characteristics between a Sphere and a Flat Plate: Rabinowitsch Model
}

\author{
Udaya P. Singh and Ram S. Gupta \\ Department of Applied Science and Humanities, Kamla Nehru Institute of Technology, Sultanpur 228118, India \\ Correspondence should be addressed to Udaya P. Singh, journals4phd@gmail.com
}

Received 22 August 2012; Accepted 29 November 2012

Academic Editor: J. Paulo Davim

Copyright (๑) 2012 U. P. Singh and R. S. Gupta. This is an open access article distributed under the Creative Commons Attribution License, which permits unrestricted use, distribution, and reproduction in any medium, provided the original work is properly cited.

\begin{abstract}
The use of additives (polyisobutylene, ethylene-propylene, lithium hydroxy stearate, hydrophobic silica, etc.) changes lubricants' rheology due to which they show pseudoplastic and dilatant nature, which can be modelled as cubic stress fluid model (Rabinowitsch fluid model). The present theoretical analysis investigates the effects of non-Newtonian pseudoplastic and dilatant lubricants on the squeezing characteristics of a sphere and a flat plate. The modified Reynolds equation has been derived and an asymptotic solution for film pressure is obtained. The results for the film pressure distribution, load carrying capacity, and squeezing time characteristics have been calculated for various values of pseudoplastic parameter and compared with the Newtonian results. These characteristics show a significant variation with the non-Newtonian pseudoplastic and dilatant behavior of the fluids.
\end{abstract}

\section{Introduction}

Squeeze film between a sphere and plate is observed in various machine elements such as ball bearings, cam and followers, and gears. The mechanical action (squeezing, shearing, etc.) leading to the generation of high pressure at the contacts [1-4] changes the rheology of the lubricants such as viscosity and density which account for the performance characteristics of machine elements. Dowson [5], Wada and Hayashi [6], and Yadav and Kapur [7] emphasized the variation of viscosity and density with temperature and pressure and reported significant changes in bearing characteristics. Denn [8], Rajagopal [9], and Renardy [10] indicated that in high pressure lubrication applications, the variation of viscosity becomes more important than the density. Variation of the viscosity also causes the instability of the lubricants' nature by changing its shearing stress-strain rate relation due to which the estimated characteristics of lubricated contacts such as sphere-plate contacts (point contacts) may deviate from the desired value. This situation is avoided by enhancing the efficiency of stabilizing properties of lubricants by the addition of additives (polyisobutylene, ethylene propylene, etc.). The use of additives minimizes the sensitivity of the lubricant to the change in the shearing strain rate and the lubricants behave like non-Newtonian pseudoplastic, dilatant, and viscoplastic fluids depending on the nature and quantity of the additives. To account for the effects of lubricant additives on the performance characteristics of lubricated point contacts, various non-Newtonian fluid models like power law, micropolar, and couple stress fluid models have been studied by researchers from time to time [11-14]. Among these fluid models, Rabinowitsch fluid model [6] is an established model to predict the effects of additives on the performance characteristics of the lubricated bearings. The shearing stress-strain relation in this model for one-dimensional fluid flow is given by

$$
\bar{\tau}_{r z}+\overline{\kappa \tau}_{r z}^{3}=\bar{\mu} \frac{\partial \bar{u}}{\partial \bar{z}},
$$

where $\bar{\mu}$ is the initial viscosity of lubricant and $\bar{\kappa}$ is the nonlinear factor responsible for the non-Newtonian effects of the fluid which will be referred to as the coefficient of pseudoplasticity. This model can be applied to Newtonian, dilatant, and pseudoplastic lubricants for $\bar{\kappa}=0, \bar{\kappa}<0$, and $\bar{\kappa}>0$, respectively. The advantage of this model lies in the fact that the theoretical analysis for the present model 


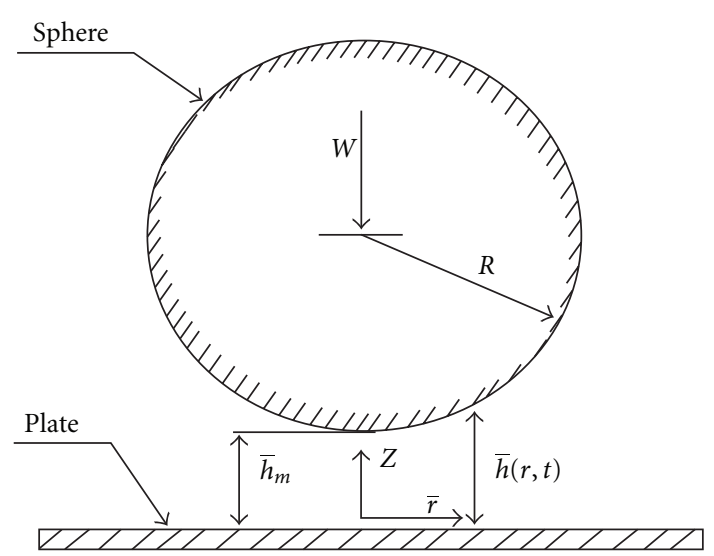

FIGURE 1: Schematic diagram of squeeze film between a sphere and a plate.

was verified with the experimental justification by Wada and Hayashi [6]. They used spindle oil as the base lubricant and concluded a decrease of dimensionless film pressure with the increase of additive (polyisobutylene). Afterwards, the theoretical study of bearing performance with nonNewtonian lubricants using this models was done by Bourgin and Gay [15] on journal bearing, Hashimoto and Wada [16] on circular plates bearing, and Lin [17] on parallel circular plates. Recently Singh et al. [18-21] used this model to study the performance characteristics of hydrostatic thrust bearings and slider bearings.

The objective of this paper is to extend the results [18-21] to squeeze film characteristics between a sphere and a plate by introducing a quantitative analysis using Rabinowitsch fluid model, which accounting for the effect of additives in the lubricant. The modified Reynolds equation governing the squeeze film pressure is derived. Squeeze film characteristics such as film pressure, load-carrying capacity, and squeezing time are presented. The importance of the present analysis lies in that the earlier theoretical investigations on sphere-plate squeezing [11-14] are based on couple stress or micropolar fluid models, which suffer the scarcity of experimental verification.

\section{Constitutive Equations and Boundary Conditions}

The physical configuration of a sphere-plate system is shown in Figure 1. The sphere is approaching towards the plate with a normal velocity $(-d \bar{h} / d \bar{t})$, separated by a lubricant thin film. The lubricant in the system is taken as a non-Newtonian Rabinowitsch fluid. The body forces and body couples are assumed to be absent.

Under the assumptions of hydrodynamic lubrication applicable to thin film as considered by Dowson [5], the field equations governing the one-dimensional motion of an incompressible non-Newtonian Rabinowitsch fluid model in polar coordinates $(r, \theta, z)$ system [18] are

$$
\frac{1}{\bar{r}} \frac{\partial}{\partial \bar{r}}(\overline{r u})+\frac{\partial \bar{w}}{\partial \bar{z}}=0
$$

$$
\begin{gathered}
\frac{\partial \bar{p}}{\partial \bar{r}}=\frac{\partial \bar{\tau}_{r z}}{\partial \bar{z}}, \\
\frac{\partial \bar{p}}{\partial \bar{z}}=0
\end{gathered}
$$

which are solved under the no-slip boundary conditions:

$$
\begin{gathered}
\bar{u}=0, \quad \bar{w}=0, \quad \text { at } \bar{z}=0, \\
\bar{u}=0, \quad \bar{w}=-\frac{\partial \bar{h}}{\partial \bar{t}}, \quad \text { at } \bar{z}=\bar{h},
\end{gathered}
$$

where $\bar{u}$ and $\bar{w}$ are the velocity components in $\bar{r}$ and $\bar{z}$ directions, respectively, and $\bar{h}$ is the film thickness between the sphere and plate.

\section{Analysis}

Integrating (3) with respect to $\bar{z}$ under boundary conditions (5) and using (1), the expression for velocity $\bar{u}$ is obtained as

$$
\begin{aligned}
\bar{u}=\frac{1}{2 \bar{\mu}}[ & \frac{\partial \bar{p}}{\partial \bar{r}} \bar{z}(\bar{z}-\bar{h})+\bar{\kappa}\left(\frac{\partial \bar{p}}{\partial \bar{r}}\right)^{3} \\
& \left.\times\left(\frac{1}{2} \bar{z}^{4}-\bar{z}^{3} \bar{h}+\frac{3}{4} \bar{z}^{2} \bar{h}^{2}-\frac{1}{4} \bar{z} \bar{h}^{3}\right)\right] .
\end{aligned}
$$

Integrating (2) with respect to $\bar{z}$ under the relevant boundary conditions (5) for $\bar{w}$ and using (6), the modified Reynolds equation is obtained as

$$
\frac{1}{\bar{r}} \frac{\partial}{\partial \bar{r}}\left[\bar{r}\left\{\bar{h}^{3} \frac{\partial \bar{p}}{\partial \bar{r}}+\frac{3 \bar{\kappa} \bar{h}^{5}}{20}\left(\frac{\partial \bar{p}}{\partial \bar{r}}\right)^{3}\right\}\right]=-12 \bar{\mu} \frac{\partial \bar{h}}{\partial \bar{t}} .
$$

In the limiting case of $\kappa \rightarrow 0$, (7) reduces to the Newtonian form of Reynolds equation obtained by Conway and Lee [22]:

$$
\frac{1}{\bar{r}} \frac{\partial}{\partial \bar{r}}\left[\bar{r}^{3} \frac{\partial \bar{p}}{\partial \bar{r}}\right]=-12 \bar{\mu} \frac{\partial \bar{h}}{\partial \bar{t}} .
$$

The expression for film thickness between the sphere and plate at a time $\bar{t}$ is taken of the form [1]

$$
\bar{h}=\bar{h}_{m}+\frac{\bar{r}^{2}}{2 R},
$$

where $R$ denote the radius of the sphere. In case of squeezing between two spheres, the value of the radius $R$ can be taken as (i) $R^{-1}=R_{1}^{-1}+R_{2}^{-1}$ for external contact and (ii) $R^{-1}=$ $R_{1}^{-1}-R_{2}^{-1}$ for internal contact, where $R_{1}$ and $R_{2}$ are the radii of the spheres.

The modified Reynolds equation (7) takes the dimensionless form:

$$
\frac{1}{r} \frac{d}{d r}\left[r\left\{h^{3} \frac{d p}{d r}+\frac{3 \alpha h^{5}}{20}\left(\frac{d p}{d r}\right)^{3}\right\}\right]=-\frac{12}{\beta},
$$

where $p=\bar{h}_{m o}^{2} \bar{p} / \bar{\mu} R\left(d \bar{h}_{m} / d \bar{t}\right)$ is the dimensionless pressure, $\alpha=\bar{\kappa}\left(\bar{\mu} R\left(d \bar{h}_{m} / d \bar{t}\right) / \bar{h}_{m o}^{2}\right)^{2}$ is the parameter of pseudoplasticity, $\beta=\bar{h}_{m o} / R$ is the sphere parameter, $h_{m}=\bar{h}_{m} / \bar{h}_{m o}$ is the 
minimum film thickness at a time $t$, and $\bar{h}_{m o}$ is the minimum film thickness at $\bar{t}=0$. The value of the pseudoplastic coefficient $\bar{\kappa}$ depends on the type of and the quantity of additives which can be determined experimentally [6]. Thus, the values of $R, \bar{h}_{m o}$, and $\bar{\mu}$ being known for a particular bearing and lubricant and the vales of $\alpha$ can be calculated with the appropriate value of $\bar{\kappa}$. However, for the validity of the present analysis, the value of $\alpha$ is restricted to $|\alpha|<0.01$.

As (10) is a nonlinear equation in $p$, it is not easy to solve it using analytical methods. Therefore, the classical perturbation method is used to solve it. The perturbation series for $p$ can be expressed in the form:

$$
p=p_{o}+\alpha p_{1}+\alpha^{2} p_{2}+\cdots .
$$

For $\alpha \ll 1$, it is sufficient, for analysis, to consider the first order term in $\alpha$ as follows:

$$
p=p_{o}+\alpha p_{1} .
$$

For the higher values of $\alpha$, second and higher order terms can be considered to increase the accuracy of the results. However, for the higher values of $\alpha$, it will be more appropriate to adopt a numerical solution procedure such as the finite element method to solve the Reynolds equation.

Substituting (12) in (10), the perturbation equations are obtained as

$$
\begin{gathered}
\frac{1}{r} \frac{d}{d r}\left[r h^{3} \frac{d p_{o}}{d r}\right]=-\frac{12}{\beta}, \\
\frac{1}{r} \frac{d}{d r}\left[r\left\{h^{3} \frac{d p_{1}}{d r}+\frac{3 h^{5}}{20}\left(\frac{d p_{o}}{d r}\right)^{3}\right\}\right]=0 .
\end{gathered}
$$

Solving (13) under the boundary conditions

$$
\frac{d p}{d r}=0 \quad \text { at } r=0, \quad p=0 \quad \text { at } r=1,
$$

the dimensionless pressure developed in the film region is:

$$
\begin{aligned}
p= & 12 \beta^{2}\left[\frac{1}{\left(1+2 h_{m} \beta\right)^{2}}-\frac{1}{\left(r^{2}+2 h_{m} \beta\right)^{2}}\right] \\
& -\frac{3456}{25} \alpha \beta^{4}\left[\frac{3+h_{m} \beta}{\left(1+2 h_{m} \beta\right)^{6}}-\frac{3 r^{2}+h_{m} \beta}{\left(r^{2}+2 h_{m} \beta\right)^{6}}\right] .
\end{aligned}
$$

3.1. Load Carrying Capacity. The load carrying capacity can be obtained by integrating the film pressure over the squeezing film area as follows:

$$
\bar{w}=2 \pi \int_{0}^{R} \bar{p} \bar{r} d \bar{r}
$$

which takes the dimensionless form:

$$
w=\int_{0}^{1} p r d r
$$

where

$$
w=\bar{w}\left[\frac{\bar{h}_{m o}^{2}}{\left(2 \pi \bar{\mu} R^{3}\left(d \bar{h}_{m} / d \bar{t}\right)\right)}\right] .
$$

3.2. Squeezing Time. The squeezing time can be calculated by integrating (18) with respect to $t$ under the condition that $h_{m}=1$ at $t=0$ as follows:

$$
t=\int_{h_{f}}^{1} w d h=\int_{h_{f}}^{1} \int_{0}^{1} r p d r d h_{m},
$$

where

$$
t=\frac{\bar{w} \bar{h}_{m o}}{2 \pi \bar{\mu} R^{3}} \bar{t} .
$$

\section{Results and Discussions}

Based on the Rabinowitsch fluids model, the effects of non-Newtonian rheology on the squeeze-film characteristics between a sphere and a plate are investigated using a dimensionless parameter $\alpha$ which accounts for the nonNewtonian nature of the lubricant, that is, for the induced nature due to the use of additives. The parameters $\alpha=0$, $\alpha<0$, and $\alpha>0$ describe the Newtonian, dilatant, and pseudoplastic lubricants, respectively. For the validity of the analysis, the numerical results for non-Newtonian lubricants are compared with the Newtonian results [12].

In order to analyze the non-Newtonian effects of fluids on the squeeze-film performance of sphere-plate system, various squeeze-film characteristics are presented with the following values:

(i) pseudoplastic parameter $\alpha=-0.01$ to 0.01 , [2, 18];

(ii) sphere parameter $\beta=0.03,0.05,[12,14]$.

Figure 2 shows the variation of dimensionless film pressure $(p)$ with respect to the dimensionless coordinate $(r)$. It is clear from the figure that the pressure is the maximum at $r=0$, that is, at the minimum film thickness and decreases towards the outer of the sphere and hence, the analysis obeys the basic theory of film pressure in sphereplate system. Again, the pressure for dilatant lubricants $(\alpha<$ 0 ) is higher than the pressure for Newtonian lubricants whereas the pressure for pseudoplastic lubricants $(\alpha>0)$ is lower than the pressure for Newtonian lubricants. Further, the pressure increases as $\alpha$ decrease from 0.01 to -0.01 . It is observed that the effect of non-Newtonian pseudoplastic and dilatant lubricants produces a remarkable change in the film pressure near $r=0$, and it decreases towards periphery $r=1$. For design parameters $\beta=0.05$ and $h_{m}=0.7$, a small value of pseudoplastic parameter $\alpha=-0.01$ (dilatant fluids) increases the film pressure by nearly $25 \%$ at $r=0.1$ and $3 \%$ at $r=0.5$. For the same design parameters, the value of pseudoplastic parameter $\alpha=0.01$ (pseudoplastic fluids) decreases the film pressure by nearly $30 \%$ at $r=0.1$ and $2 \%$ at $r=0.5$. It shows that the pseudoplastic and dilatant lubricants produce larger effects with the higher film pressure.

Figure 3 shows the variation of dimensionless maximum film pressure $\left(p_{\max }\right)$ with respect to the dimensionless minimum film thickness $\left(h_{m}\right)$. The effect of dilatant lubricants is observed to increase the maximum film pressure from 


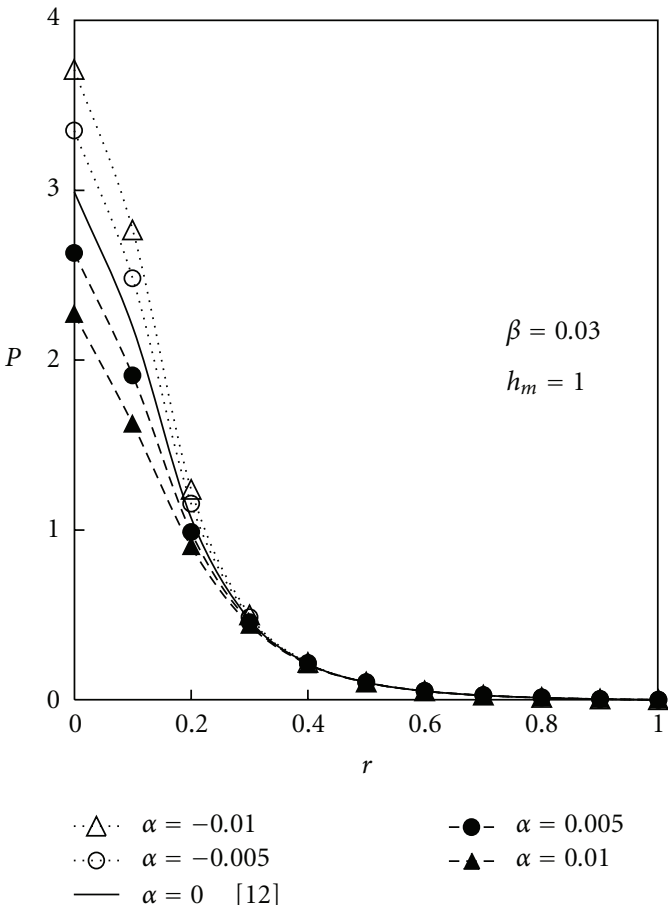

(a) $\beta=0.03, h_{m}=1$

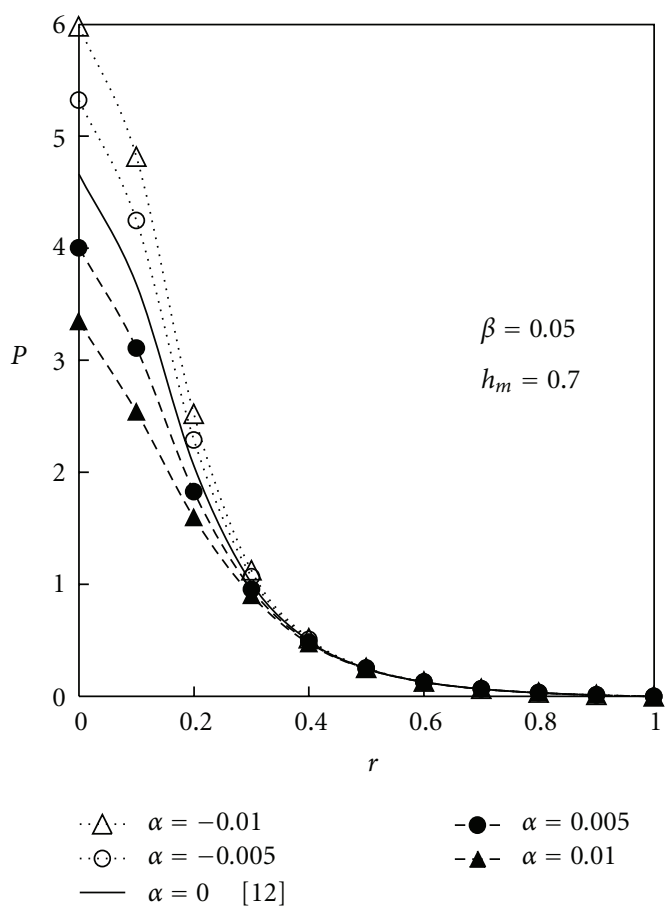

(b) $\beta=0.05, h_{m}=0.70$

FIGURE 2: Variation of dimensionless pressure with respect to the dimensionless radius $r$ for different values of pseudoplastic parameter $\alpha$.

its value in Newtonian case whereas the effect of pseudoplastic lubricant decreases the maximum film pressure from its value in the Newtonian case. Furthermore, the maximum pressure increases with the decrease of pseudoplastic parameter $\alpha$ from 0.01 to -0.01 . There is also a relative change in the maximum pressure for pseudoplastic and dilatant lubricants, which increases with the decrease of film thickness. For the sphere parameter $\beta=0.05$, a small value of pseudoplastic parameter $\alpha=-0.01$ increases the maximum film pressure by nearly $12 \%$ to $25 \%$ as the minimum film thickness $h_{m}$ decreases from 1 to 0.7 . For the same value of $\beta$ and $h_{m}$, the value of pseudoplastic parameter $\alpha=0.01$ (pseudoplastic fluids) decreases the film pressure nearly $15 \%$ to $30 \%$. Therefore, it can be safely said that the higher the film pressure, the greater the change produced by pseudoplastic and dilatant lubricants is.

Figure 4 shows the variation of dimensionless load carrying capacity $(W)$ of the system with respect to the dimensionless minimum film thickness $\left(h_{m}\right)$. It is observed that the load capacity obtained with dilatant lubricants is higher than that with Newtonian lubricants, and the load capacity obtained with pseudoplastic lubricants is lower than its value obtained with Newtonian lubricants. Furthermore, the load capacity increases with the decrease of pseudoplastic parameter $\alpha$ from 0.01 to -0.01 . It is also observed that there is a relative change in load capacity obtained with the different values of pseudoplastic parameter, which increases with the decrease of film thickness. For the sphere parameter $\beta=0.05$, the effect of dilatant lubricant $\alpha=-0.01$ increases the load capacity by $10 \%$ to $15 \%$ as the minimum film thickness $h_{m}$ decreases from 1 to 0.7 and for the same value of $\beta$ and $h_{m}$, the effect of pseudoplastic lubricants $\alpha=0.01$ decreases the film pressure from $15 \%$ to $20 \%$.

Figure 5 shows the time $(t)$ elapsed in squeezing the film from its initial thickness $h_{m}=1$ to a final thickness $h_{m}=h_{f}$. It is observed that for each value of $h_{f}$ and $\beta$, the squeeze time for dilatant lubricants is longer than that with the Newtonian lubricants, whereas, the squeeze time for pseudoplastic lubricants is less than its value in the Newtonian case. Further, the squeeze time increases with the decrease of pseudoplastic parameter from 0.01 to -0.01 . This phenomenon can be interpreted as a result of the increase in the film pressure from pseudoplastic to dilatant lubricants. For the sphere parameter $\beta=0.03$, the time to squeeze the film to $h_{f}=0.7$ is increased by nearly $23 \%$ with dilatant lubricant $\alpha=-0.01$ in comparison with the Newtonian case, whereas the same is decreased by nearly $18 \%$ for pseudoplastic lubricants. For the sphere parameter $\beta=0.05$, the time to squeeze the film from $h=1$ to $h=0.7$ is increased by nearly $14 \%$ with dilatant lubricant $\alpha=-0.01$ in comparison with the Newtonian case. For the same value of $\beta$ and $h_{f}$, the squeeze time is reduced by nearly $16 \%$ with pseudoplastic lubricants $\alpha=0.01$. Thus, dilatant lubricants increase and pseudoplastic lubricants reduce the squeeze time of the bearing.

\section{Conclusions}

Based on the Rabinowitsch fluid model (cubic stress model) for non-Newtonian pseudoplastic and dilatant fluids, the 


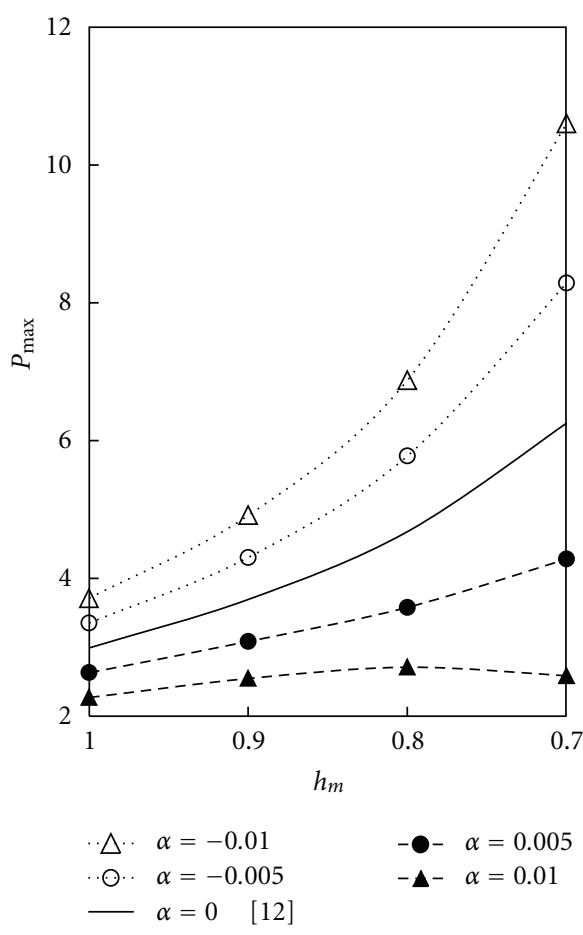

(a) $\beta=0.03$

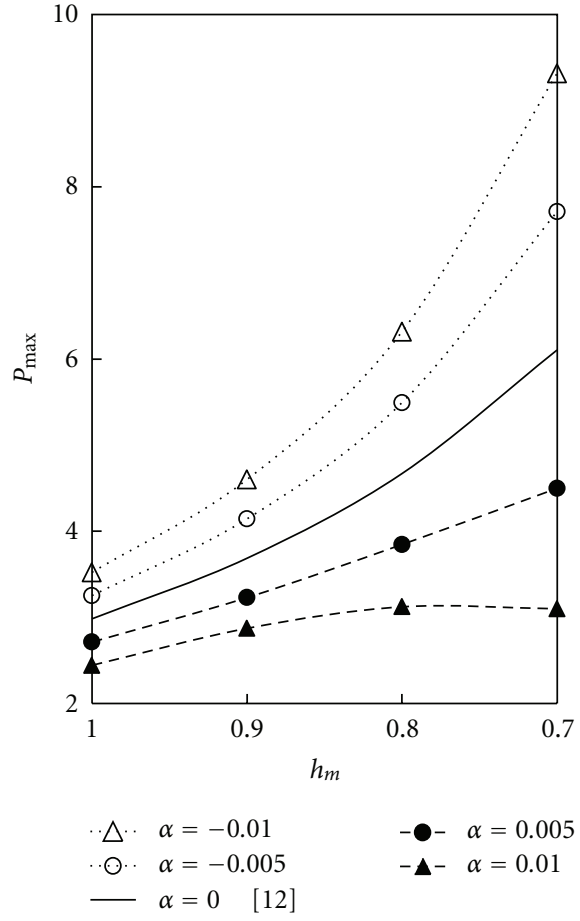

(b) $\beta=0.05$

FIGURE 3: Variation of the dimensionless maximum film pressure with respect to the minimum film thickness $h_{m}$ for different values of parameter $\alpha$.

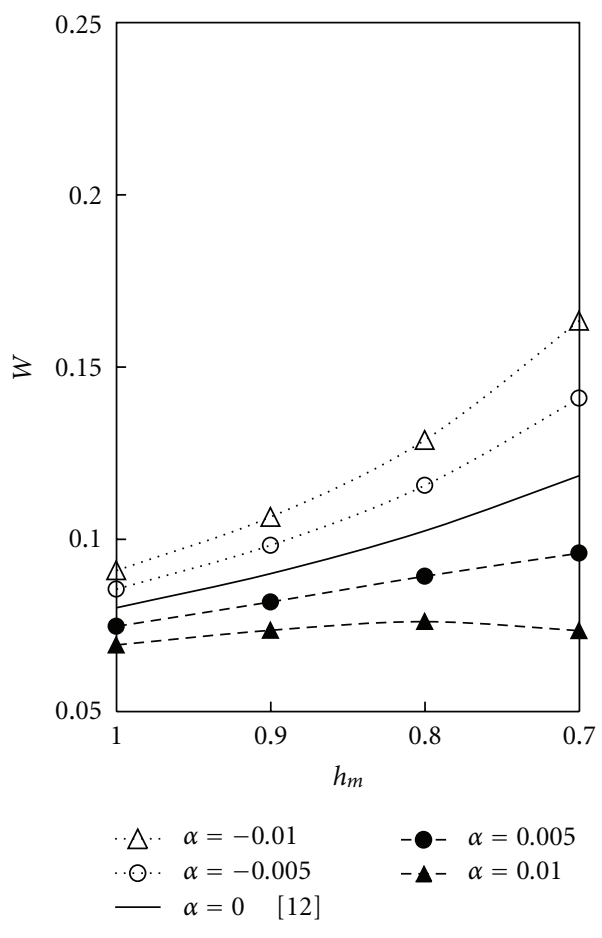

(a) $\beta=0.03$

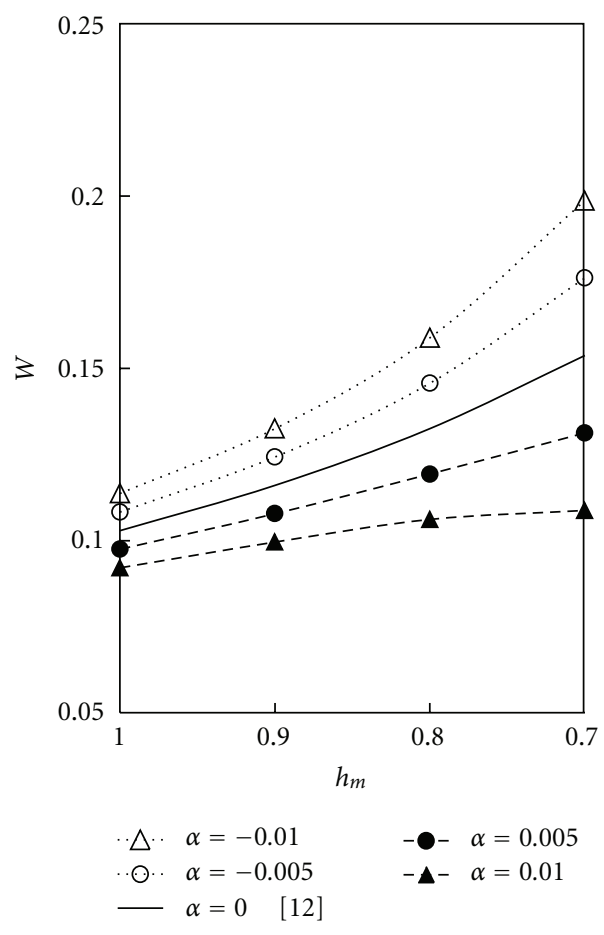

(b) $\beta=0.05$

FIGURE 4: Variation of the dimensionless load capacity with respect to the dimensionless minimum film thickness $h_{m}$ for values of parameter $\alpha$. 


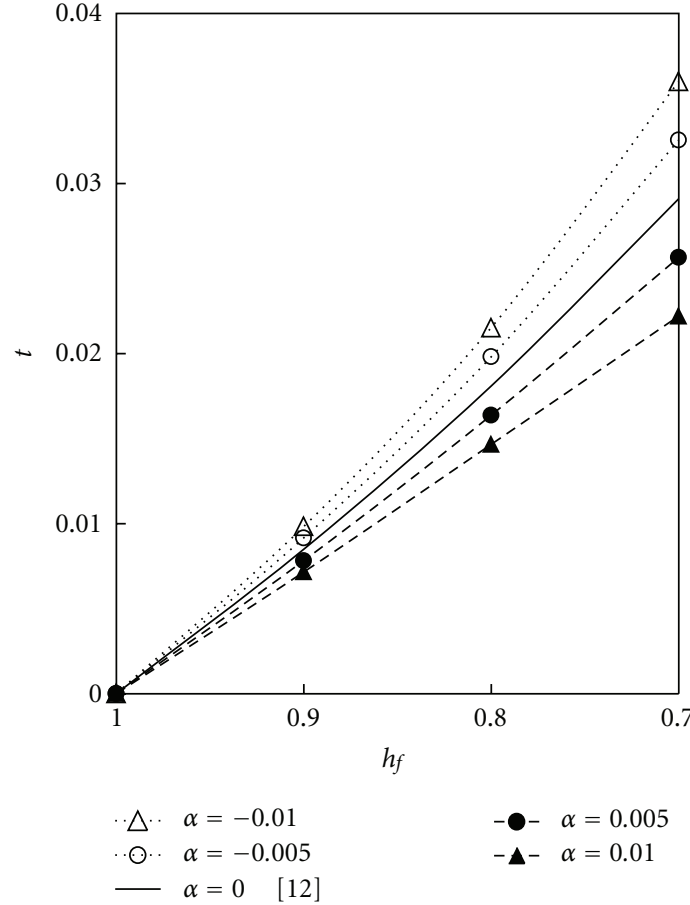

(a) $\beta=0.03$

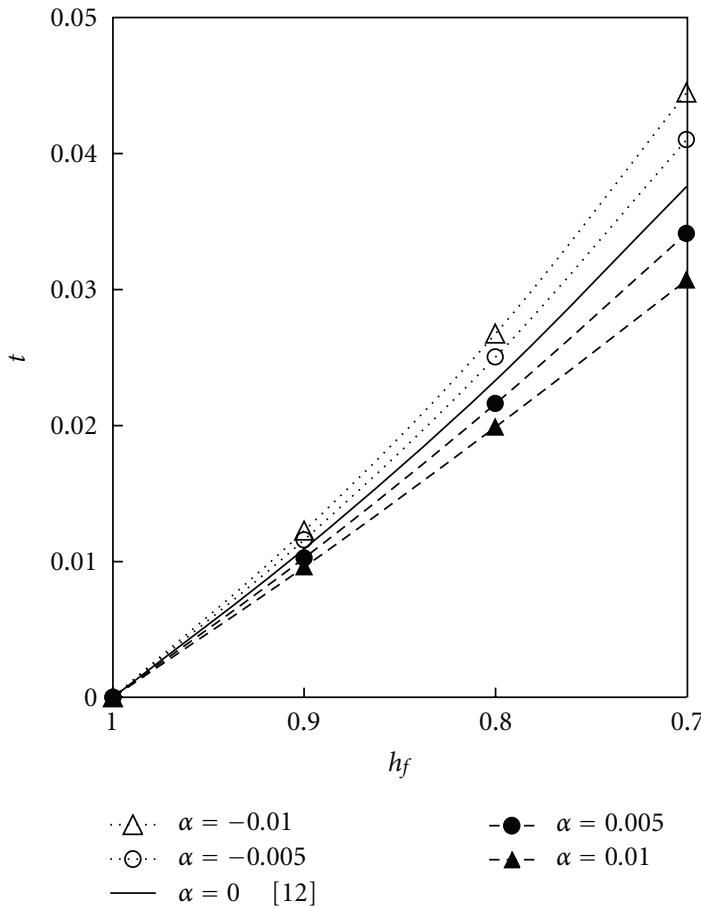

(b) $\beta=0.05$

FIGURE 5: Variation of dimensionless squeeze time with respect to the squeezed film thickness $h_{f}$ for different values of parameter $\alpha$.

effects of lubricant additives on the performance characteristics of squeezing film between a sphere and a plate are presented avoiding the inertia and cavitation effects. The analytical solution for pressure distribution is obtained using a classical perturbation technique. Based on the present theoretical analysis, the following results have been drawn.

(1) Dilatant lubricants increase the pressure and load carrying capacity significantly, whereas the case is reversed with the pseudoplastic lubricants.

(2) On comparing with the Newtonian case, dilatant lubricants increase the squeeze time, whereas the pseudoplastic lubricants decrease it.

(3) As the squeezing time of the sphere-plate system is significantly increased with the dilatant lubricants, it is expected that the use of additives can reduce the vibration in the sphere-plate systems.

Thus, the present analysis can also provide a guideline to control the vibration in the system. Hence, the results are expected to be more helpful for better bearing performance and stability. However, an experimental validation of these results is required at laboratory level.

\section{Nomenclature}

- : $\quad$ Bar denotes the dimensional quantities

$\bar{h}, h: \quad$ Film thickness defined in (9), $h=\bar{h} / h_{m o}$

$\bar{h}_{m}, h_{m}$ : Minimum film thickness, $h_{m}=\bar{h}_{m} / \bar{h}_{m o}$ $\bar{h}_{m o}: \quad$ Initial minimum film thickness

$\bar{p}, p: \quad$ Film pressure, $p=\bar{h}_{m o}^{2} \bar{p} / \bar{\mu} R\left(d \bar{h}_{m} / d \bar{t}\right)$

$p_{o}, p_{1}$ : Dimensionless perturbed film pressures

$\bar{r}, r: \quad$ Radial coordinate, $r=\bar{r} / R$

$R: \quad$ Radius of sphere

$\bar{t}, t: \quad$ Time, $t=\left(\bar{W} \bar{h}_{m o} / 2 \bar{\mu} B R^{2}\right) \bar{t}$

$\bar{u}, \bar{w}: \quad$ Components of velocity

$\bar{W}, W$ : Load capacity, $\quad W \quad=$

$\left(h_{m o}^{2} /\left(2 \bar{\mu} B R^{2}\left(d \bar{h}_{m} / d \bar{t}\right)\right)\right) \bar{W}$

$\alpha: \quad \bar{\kappa}\left[\bar{\mu} R\left(d \bar{h}_{m} / d \bar{t}\right) / \bar{h}_{m o}^{2}\right]^{2}$

$\beta: \quad$ Design parameter $\left(\bar{h}_{m o} / R\right)$

$\bar{\kappa}: \quad$ Coefficient pseudoplasticity

$\bar{\mu}: \quad$ Viscosity of lubricant

$\bar{\tau}_{r z}: \quad$ Stress component.

\section{Acknowledgments}

The authors, hereby, thank Dr. M. Fillon (Director of Research, Centre National de la Recherche Scientifique, University of Poitiers) and Dr. V. K. Kapur (Former Professor and Chairman, KNIT, Sultanpur, India) for providing useful materials and guidelines to enhance the content of the paper.

\section{References}

[1] M. D. Pascovici, C. S. Popescu, and V. G. Marian, "Impact of a rigid sphere on a highly compressible porous layer imbibed with a Newtonian liquid," Proceedings of the Institution of Mechanical Engineers J, vol. 224, no. 8, pp. 789-795, 2010. 
[2] L. E. Goodman and G. E. Bowie, "Experiments on damping at contacts of a sphere with flat plates-Test results for the constant normal force/varying tangential force problem are compared with theoretical predictions," Experimental Mechanics, vol. 1, no. 2, pp. 48-54, 1961.

[3] H. A. Kalameh, A. Karamali, C. Anitescu, and T. Rabczuk, "High velocity impact of metal sphere on thin metallic plate using smooth particle hydrodynamics (SPH) method," Frontiers of Structural and Civil Engineering, vol. 6, no. 2, pp. 101-110, 2012.

[4] G. H. Meeten, "Squeeze flow between plane and spherical surfaces," Rheologica Acta, vol. 40, no. 3, pp. 279-288, 2001.

[5] D. Dowson, "Inertia effects in hydrostatic thrust bearings," Journal of Basic Engineering, vol. 83, no. 2, pp. 227-234, 1961.

[6] S. Wada and H. Hayashi, "Hydrodynamic lubrication of journal bearings by pseudo- plastic lubricants," Bulletin of JSME, vol. 14, no. 69, pp. 279-286, 1971.

[7] J. S. Yadav and V. K. Kapur, "On the viscosity variation with temperature and pressure in thrust bearing," International Journal of Engineering Science, vol. 19, no. 2, pp. 269-277, 1981.

[8] M. M. Denn, Polymer Melt Processing, Cambridge University Press, Cambridge, UK, 2008.

[9] K. R. Rajagopal, "On implicit constitutive theories for fluids," Journal of Fluid Mechanics, vol. 550, pp. 243-249, 2006.

[10] M. Renardy, "Parallel shear flows of fluids with a pressuredependent viscosity," Journal of Non-Newtonian Fluid Mechanics, vol. 114, no. 2-3, pp. 229-236, 2003.

[11] J. R. Gregory, "Squeeze film between two spheres in a powerlaw fluid," Journal of Non-Newtonian Fluid Mechanics, vol. 63, no. 2-3, pp. 141-152, 1996.

[12] J. R. Lin, L. J. Liang, and L. M. Chu, "Effects of non-Newtonian micropolar fluids on the squeeze-film characteristics between a sphere and a plate surface," Proceedings of the Institution of Mechanical Engineers J, vol. 224, no. 8, pp. 825-832, 2010.

[13] N. B. Naduvinamani, P. S. Hiremath, and G. Gurubasavaraj, "Effect of surface roughness on the couple-stress squeeze film between a sphere and a flat plate," Tribology International, vol. 38, no. 5, pp. 451-458, 2005.

[14] A. A. Elsharkawy and K. J. Al-Fadhalah, "Squeeze film characteristics between a sphere and a rough porous flat plate with micropolar fluids," Lubrication Science, vol. 23, no. 1, pp. $1-18,2011$.

[15] P. Bourgin and B. Gay, "Determination of the load capacity of a finite width journal bearing by a finite-element method in the case of a non-newtonian lubricant," Journal of Tribology, vol. 106, no. 2, pp. 285-290, 1984.

[16] H. Hashimoto and S. Wada, "Effects of fluid inertia forces in parallel circular squeeze film bearings lubricated with pseudoplastic fluids," Journal of Tribology, vol. 108, no. 2, pp. 282-287, 1986.

[17] J. R. Lin, "Non-newtonian effects on the dynamic characteristics of one-dimensional slider bearings: rabinowitsch fluid model," Tribology Letters, vol. 10, no. 4, pp. 237-243, 2001.

[18] U. P. Singh, R. S. Gupta, and V. K. Kapur, "On the steady performance of hydrostatic thrust bearing: rabinowitsch fluid model," Tribology Transaction, vol. 54, no. 5, pp. 723-729, 2011.

[19] U. P. Singh, R. S. Gupta, and V. K. Kapur, "Effects of inertia in the steady state pressurised flow of a non-newtonian fluid between two curvilinear surfaces of revolution: rabinowitsch fluid model," Chemical and Process Engineering, vol. 32, no. 4, pp. 333-349, 2011.
[20] U. P. Singh, R. S. Gupta, and V. K. Kapur, "On the steady performance of annular hydrostatic thrust bearing: rabinowitsch fluid model," Journal of Tribology, vol. 134, no. 4, Article ID 044502, 5 pages, 2012.

[21] U. P. Singh, R. S. Gupta, and V. K. Kapur, "On the performance of pivoted curved slider bearings: rabinowitsch fluid model," Tribology in Industry, vol. 34, no. 3, pp. 127-136, 2012.

[22] H. D. Conway and H. C. Lee, "Impact of a lubricated surface by a sphere," Journal of Tribology, vol. 97, no. 4, pp. 613-615, 1975. 

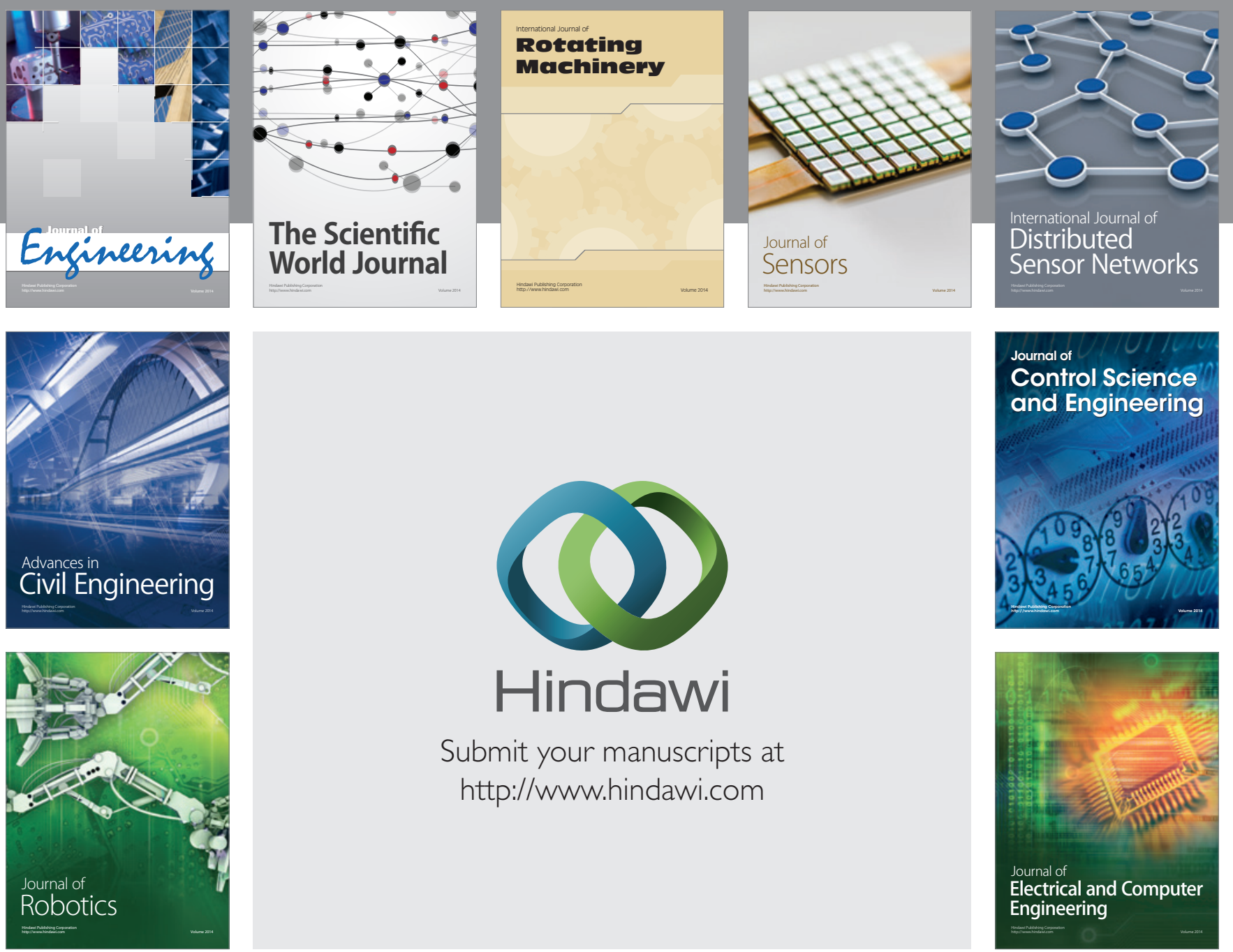

Submit your manuscripts at

http://www.hindawi.com
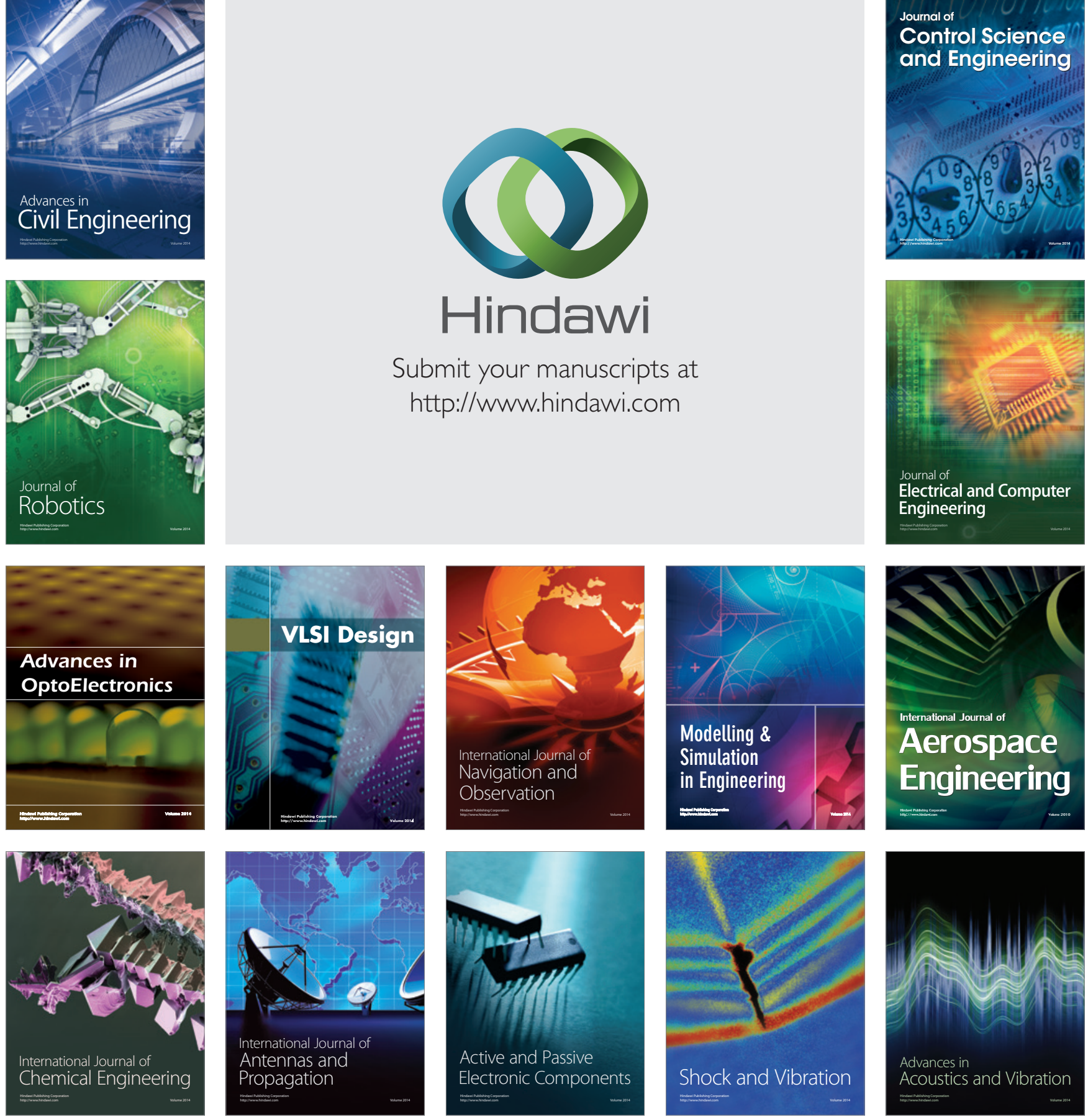